\title{
ASSOCIATIVE MEMORY COMPARISON FOR TARGET IDENTIFICATION
}

\author{
David W. Banton and Mark A. Mehalic \\ Department of Electrical and Computer Engineering \\ Air Force Institute of Technology \\ Wright-Patterson Air Force Base, Ohio 45433-6583
}

\begin{abstract}
This paper presents the execution speed comparison of two associative memory organizations used to identify weapon platforms from intercepted radar emanations. The two associative memory organizations were chosen because of their fundamentally different levels of processing granularity. The CAM was chosen because its processing is performed within the memory cell, while the bit-serial wordparallel associative memory was chosen because its processing is performed at the memory word level. Both organizations were designed, modelled, and simulated to determine which was the faster in comparing 8-bit, and 32-bit target parameters.

The associative memories were designed to store the parameters of at least 72 weapon platforms, with each platform consisting of six parameters, and each parameter consisting of an upper and lower limit. The number of bits per word and the number of words within the memory were varied to accommodate the target parameters and to enhance the comparison speed. The comparison speed was calculated by summing the critical path delays through each memory. The comparison speed results indicate which associative memory organization is the more appropriate for identifying the weapon platform.
\end{abstract}

Introduction

Aircraft flying in a hostile environment can expect to intercept radar emanations from potentially lethal weapon platforms. The aircraft's survival depends upon quick and accurate identification of the weapon platform emitting the radar signal followed by an appropriate response.

In general, an aircraft's computers begin the weapon platform identification process by measuring key parameters of the intercepted radar emanation. Once the parameters are available, they are compared to the previously measured parameters of known weapon platforms. If the comparison yields a match, the threat is identified, otherwise the parameters are stored for further analysis.
For the comparison process, speed is essential. The time to measure each intercepted radar emanation parameter varies, so to accelerate the comparison, the process can begin with the first available parameter and can be completed as more parameters become available. Since the parameter values of a weapon platform radar emanation may vary, the aircraft computers compare the intercepted radar emanation parameters to a range of acceptable values determined from known weapon platforms, which implies that each previously measured parameter consists of an upper and lower limit.

The weapon platform identification process resembles a database search, where the intercepted radar emanation parameters form the comparand, the previously measured parameter limits of known weapon platforms form the database, and the comparison is the operator.

The target identification process and its database analogy exhibit several attributes that make it well suited for storage within an associative memory. The target identification process requires quick comparisons and potentially an associative memory can fulfill that requirement [1]. The database contents do not change once initially loaded, though it may be appended with the parameters of unidentifiable radar emanations. This means that the associative memory size should support the database size for the duration of the mission. The number of bits per parameter limit is a constant and is not exceedingly large. This allows an orderly partitioning of the associative memory.

\section{The Associative Memory Comparison}

This paper compares two types of associative memory organizations to determine which is the more appropriate for target identification. The two associative memory organizations were chosen because of their fundamentally different levels of processing granularity. The CAM was chosen because its processing is performed within the memory cell, while the bit-serial word-parallel associative memory was chosen because its processing is performed at the memory word level. 
This paper examines a database consisting of 72 weapon platforms (plus two spares), with each platform consisting of six parameters, and each parameter consisting of an upper and lower limit. The number of bits required to represent each limit can be either 8 or 32 . To accommodate the database, the associative memories can be organized into phrases [2]. Each phrase consists of a fixed number of words, and each word consists of a fixed number of bits. When the phrase mode is used, the results of comparing one word within a phrase is passed to the next to continue the comparison.

\section{The CAM}

Figure 1 displays the CAM organization. The CAM has a comparand register to store the data for comparison against the contents of the memory array. It has of a two dimensional array of memory cells with each cell containing the logic to perform a scalar equal-to operation with one input from the comparand and the other from the memory cell. It has a search results register to store the comparison results, or as an enhancement, the inverse of the comparison results. The contents of the masking register determine whether a bit-slice of the memory array is compared to the corresponding comparand bit. Those bit-slices not masked are compared to the comparand register contents to form a result that is stored in the search results register. The CAM has a word select register capable of selecting the entire memory or a subset of words, and has an output register to store data read from the memory array.

Data are stored into the memory either one word at a time, to a subset of words, or to the entire memory. Data are retrieved from memory one word at a time. The location of data storage and retrieval is defined by the CAM and not by the host. The CAM can execute only equivalence comparisons (equal-to or not-equal-to). To execute a comparison, the results of comparing a bit of the comparand to the corresponding bit-slice of memory are logically anded with other bit-slice comparisons to form a final result, which is stored in the search results register. Since the CAM can only execute equivalence comparisons, the data format has no effect upon the comparison procedure, so comparand bits can be compared to the corresponding bit-slice in any order, including simultaneously.

A certain portion of each word in the CAM is used for purposes other than data storage. Both word and phrase operation modes require $T_{\text {vif }}$ bits to identify the valid words. The phrase operation mode requires an additional $T_{p f}$ bits to identify the word position within a phrase.

The relationship between the equivalence comparisons and the CAM organization allows a CAM to compare the entire database against the comparand and to form the result in the time to execute one equivalence comparison, tam. In the case where phrases are used, the equivalence comparison can be executed on a phrase in a time equal to the number of associative memory words required to form a phrase, $M$, times the equivalence comparison time, $\mathrm{Mt}_{\mathrm{cam}}$, plus $(M-1) \mathfrak{t}_{\mathrm{T}}$, where $(M-1)$ is the number of transfers necessary to compare an entire phrase, and $t_{T}$ is the time required to transfer the comparison results of one word to another word in the same phrase.

The average number of equivalence comparisons required to execute a threshold comparison (greater-than, less-than, not-greater-than, and not-less-than) is derived from Table 1. The table gives the number of equivalence comparisons required to execute a threshold comparison on each element of a database with a field length ranging from 1 to 6 bits. For example, for a database field containing a single bit, it requires one equivalence comparison to find all database elements not-greater-than ' 0 ' while it does not require an equivalence comparison to find all elements notgreater-than ' 1 '. For a field containing two bits, it requires one equivalence comparison to find all elements not-greaterthan " 00 ", " 01 ", or " 10 ", though it does not require an equivalence comparison to find all database elements notgreater-than "11", etc. If the number of bits in a field equals $\mathrm{T}$, then on average, the CAM requires $((\mathrm{T}-1) / 2)+\left(1 / 2^{\mathrm{T}}\right)$ equivalence comparisons to execute a threshold comparison. As $\mathrm{T}$ becomes large $((\mathrm{T}-1) / 2)+\left(1 / 2^{\mathrm{T}}\right)$ approaches $((\mathrm{T}-1) / 2)$. Since $t_{\text {min }}$ is the time to execute an equivalence comparison, the average time to execute a threshold comparison is approximately $((\mathrm{T}-1) / 2) \mathrm{t}_{\mathrm{can}}$, and if phrases are used, the average execution time is $\mathrm{M}((\mathrm{T}-1) / 2) \mathrm{t}_{\mathrm{cm}}+(\mathrm{M}-1) \mathrm{t}_{\mathrm{T}}$. Range comparisons take (T-1) equivalence comparisons with an approximate execution time of $(\mathrm{T}-1) \mathrm{t}_{\mathrm{com}}$ in the word mode, and $M(T-1) t_{\text {man }}+(M-1) t_{T}$ in the phrase mode.

Figure 2 shows the CAM architecture. The CAM consists of four components: the data write circuit, the CAM array, the data read circuit, and the word-select circuit. The comparand and masking registers of the memory organization drive the data write circuit. The word-select register and search results register of the memory organization are found within the word-select circuit. The data read circuit drives the output register.

The data write circuit transforms the data-in (comparand), mask-in, and write input to form the data, data-not, and mask output. If the write port is enabled, then a write operation or a comparison can occur; otherwise, a read operation can occur. The word-select circuit uses the control-in stimulus to drive either the word-select or searchselect ports. If the word-select port is chosen, either a read or write operation can occur. If the search-select port is chosen, a comparison can occur. The results of a comparison are mapped back to and stored within the word-select circuit through the word-match port. The results of a write or read operation are provided to the output register by way of the data read circuit. The data read circuit combines the 
data and data-not input to form data-out. Data-out assumes the value of the comparand during a write operation or a comparison, and assumes the value of the selected word in memory during a read operation.

\section{The Bit-Serial Word-Parallel Associative Memory}

Figure 3 displays the bit-serial word-parallel associative memory. Like the CAM, it consists of a two dimensional array of memory cells. Though the memory cells cannot execute logical operations, they can be accessed as part of a word using the word address circuitry or as part of a bit-slice using the bit-slice address circuitry. Like the CAM, the bit-serial word-parallel associative memory has a comparand register and a search results register, but a mask register is not necessary.

Data are stored into and retrieved from memory one word at a time. The location of data storage and retrieval is defined by an address. Data can also be retrieved one bitslice at a time. The bit-serial word-parallel associative memory executes equivalence and threshold comparisons using the same algorithm. It operates by storing a bit of the comparand and a bit-slice of the memory into the processing element array. Once stored, the processing element array compares the comparand bit to the bit-slice of the memory and store the results in the search results register. Next another comparand bit and bit-slice of memory are stored in the processing elements, compared, and combined with the previous comparison. The process continues until every pertinent bit-slice is compared [3].

Data are stored within a field using a magnitude representation, so the first bit of a field represents the most significant digit of the data. Equivalence comparisons can start anywhere in the field because of the bit-position independence, but all other comparisons must start by first comparing the most significant bit-position and storing the results, then by comparing the second most significant bitposition, etc.

The bit-serial word-parallel associative memory words are organized like the CAM. When operating in the word mode, $T_{v a t}$ bits are required to identify the valid words, and when operating in the phrase mode, $T_{\text {vef }}$ bits are again required and $T_{p r}$ bits are required to identify the word position in a phrase.

For $\mathrm{T}$ bits in a data field, $\left(\mathrm{T}+\mathrm{T}_{\text {vdf }}\right)$ comparisons are required in the word mode, and $\mathrm{M}\left(\mathrm{T}+\mathrm{T}_{\mathrm{vec}}+\mathrm{T}_{\mathrm{p} f}\right)$ comparisons are required in the phrase mode. Using $t_{1}$ as the time to evaluate a bit-slice, then the time to execute a comparison in the word mode is $\left(T+T_{v d f}\right) t_{1}$, and for the phrase mode $M\left(T+T_{\text {vof }}+T_{p}\right) t_{1}+(M-1) t_{T}$.

Figure 4 shows the bit-serial word-parallel associative memory architecture. It consists of ten components: the data write circuit, the address drivers, the address decode circuit, the slice address drivers, the slice address decode circuit, the bit-serial array, the data read circuit, the slice data read circuit, the processing element array, and the address encoder.

The data write circuit and data read circuit perform the same function as in the CAM architecture. The address drivers and address decode circuit take the address-in stimulus to select a single word to write to or read from. The slice address drivers and slice address decode circuit select a bit-slice of the memory to read into the processing element array. The processing element array stores elements of the comparand and bit-slices of memory to perform equivalence and threshold comparisons. The results of the comparison may identify words that meet a comparison criteria. The address of the first word in memory that matches the comparison criteria is formulated using the address encoder.

\section{Associative Memory Execution of Instruction Mix}

The previous two sections defined the amount of time the CAM and the bit-serial word-parallel associative memory require to execute equivalence and threshold comparisons. This section analyzes the performance of each associative memory to determine the better selection.

Let $x$ be the percentage of equivalence comparisons and let $y$ be the percentage of threshold comparisons that an associative memory executes in a program. The variable, $y$, is calculated with the assumption that range comparisons are two threshold comparisons. This analysis assumes that $x+y=1$.

In the word mode, the CAM execution time for a mix of comparisons is:

$$
(x+((T-1) / 2) y) t_{\operatorname{mom}}=\text { avg exec time }
$$

Substituting $x=1-y$ and reorganizing yields:

$$
\left((1+((T-3) / 2) y) t_{\text {cam }}=\right.\text { avg exec time }
$$

The phrase mode requires $M$ repetitions of Equation (2) plus the time to transfer the results between words.

$$
\begin{aligned}
& M\left((1+((T-3) / 2) y) t_{\text {com }}+(M-1) t_{T}\right. \\
& =\text { avg exec time }
\end{aligned}
$$

In the word mode, the bit-serial word-parallel associative memory execution time for a mix of comparisons is:

$$
\left.(x+y)\left(T+T_{v+1}\right) t_{1}\right)=\text { avg exec time }
$$

Since $x+y=1$, the previous equation yields: 


$$
\left(\mathrm{T}+\mathrm{T}_{\mathrm{vdf}}\right) \mathrm{t}_{1}=\text { avg exec time }
$$

Once again, the phrase mode requires $M$ repetitions of Equation (5) plus the time to transfer the results between words.

$$
M\left(T+T_{v d f}+T_{p t}\right) t_{1}+(M-1) t_{T}=\text { avg exec time }
$$

To determine when the CAM is the better organization selection, suppose for the phrase mode,

$$
\begin{aligned}
& \mathrm{M}\left((1+((\mathrm{T}-3) / 2) \mathrm{y}) \mathrm{t}_{\mathrm{cam}}+(\mathrm{M}-1) \mathrm{t}_{\mathrm{T}} \leq\right. \\
& \mathrm{M}\left(\mathrm{T}+\mathrm{T}_{\mathrm{vdf}}+\mathrm{T}_{\mathrm{pf}}\right) \mathrm{t}_{1}+(\mathrm{M}-1) \mathrm{t}_{\mathrm{T}}
\end{aligned}
$$

Assuming the circuitry to transfer the results from one word to another is approximately the same for each organization. Equation (7) reduces to:

$$
(1+((\mathrm{T}-3) / 2) \mathrm{y}) \mathrm{t}_{\mathrm{com}} \leq\left(\mathrm{T}+\mathrm{T}_{\mathrm{vuf}}+\mathrm{T}_{\mathrm{pf}}\right) \mathrm{t}_{1}
$$

Equation (8) reduces to:

$$
t_{\mathrm{cam}} / t_{1} \leq 2\left(T+T_{v d r}+T_{p t}\right) /(2+(T-3) y)
$$

On the left side of Equation (9) are the variables dependent upon the architecture implementation, while on the right side are the variables dependent upon the algorithms used to execute the search.

\section{Results}

Both the CAM and the bit-serial word-parallel associative memory organizations were designed as a VLSI circuit using the architectures presented. The layouts were constructed using CMOS, single-poly, double-metal, 0.6 micro-meter lambda design rules. The layouts were extracted to SPICE files and simulated for databases consisting of $8,16,32,64$, and 128 words, and word lengths of 12,20 , and 36 bits. The critical delays for each associative memory were measured, tabulated, and summed to obtain an approximate time to read, write, and compare. For each delay, linearly increasing the number of bits in a word or the number of words in the database causes a corresponding linear increase in the read, write, and comparison delays. The delays for the various sizes of databases were calculated, to obtain an approximate value of $t_{\text {tard }} / t_{1}$, as shown in Table 2 and Table 3.

Table 2 gives the pertinent information for a $72+2$ platforms/database, consisting of 6 parameters/platform, 2 limits/parameter, and 8 bits/limit. A variety of memory organizations capable of accommodating the database are evaluated. $T$ is allowed to vary from 8 bits to 96 bits. $T_{\text {vor }}$ is assigned a single bit. The number for the words/phrase column is calculated to be of sufficient size to store the limits for a platform. $T_{p t}$ is calculated by the equation $\mathrm{T}_{\mathrm{p}}=\left[\log _{2}\right.$ (words/phrase)]. The number in the column, words/memory, is calculated to accommodate 72 platforms plus 2 spares. The variable y is set to 1 , which means every comparison is a threshold comparison. The formula, $2\left(T+T_{v a r}+T_{p t}\right) /(2+(T-3) y)$, is calculated using the previously discussed data.

The sum of the associative memory delays shows that the fastest database comparisons occur when $T$ equals 96 bits. Table 2 shows that for T equal to 96 bits, Equation (9) holds true, and the CAM is the better organization selection for threshold comparisons. The CAM is also the better choice when T equals 8 bits. Here, the CAM is the better choice not because $t_{\text {can }}$ increases at a slower rate than $t_{1}$, but rather because of the overhead necessary to compare the valid data and phrase fields for the bit-serial wordparallel associative memory.

Table 3 gives the pertinent information for a $72+2$ platforms/database, consisting of 6 parameters/platform, 2 limits/parameter, and 32 bits/limit. $T$ is allowed to vary from 32 bits to 384 bits. $T_{\text {yor }}$ the words/phrase column, $T_{p p}$, and the words/memory column, are calculated as discussed above. As before, the variable $y$ is set to 1 , and the formula, $2\left(T+T_{v d}+T_{p}\right) /(2+(T-3) y)$, is calculated using the previously discussed data.

The sum of the associative memory delays shows that the fastest database comparisons occur when $\mathrm{T}$ equals 384 bits. Table 3 shows that for $\mathrm{T}$ equal to 384 bits, Equation (9) holds true, and the CAM again is the better organization selection for threshold comparisons.

\section{Summary}

This paper presented the execution speed comparison of two associative memory organizations used to identify weapon platforms from intercepted radar emanations. The paper examined the algorithms necessary to execute equivalence and threshold comparisons, discussed the design and simulation of the two associative memory organizations, measured the two associative memory architecture delays, and compared the two memories. The results indicated that the CAM can execute threshold comparisons faster than the bit-serial word-parallel associative memory on small databases when properly organized and using the architectural designs discussed above.

\section{Acknowledgements}

This work is supported by Wright Laboratory, Electronics Warfare Division (WL/AAWP-1), WrightPatterson AFB, OH 45433.

\section{References}

[1] C. R. DiFiore and P. B. Berra, "A Quantitative Analysis of the Utilization of Associative Memories in Database Man- 
agement," IEEE Transactions on Com-

puters, vol. $C-23$, no. 2, pp. 121-132,

February 1974.

[2] T. H. Yeap, W. M. Loucks, W. M. Snelgrove, and S. G. Zaky, "Implementing the Vastor Architecture Using a VLSI Array of 1-Bit Processors," IEEE International Conference on Computer Design, pp. 494499, 1985.

[3] L. Chisvin, and R. Duckworth, "ContentAddressable and Associative Memory: Alternatives to the Ubiquitous RAM," Computer, pp. 51-67, July 1989.
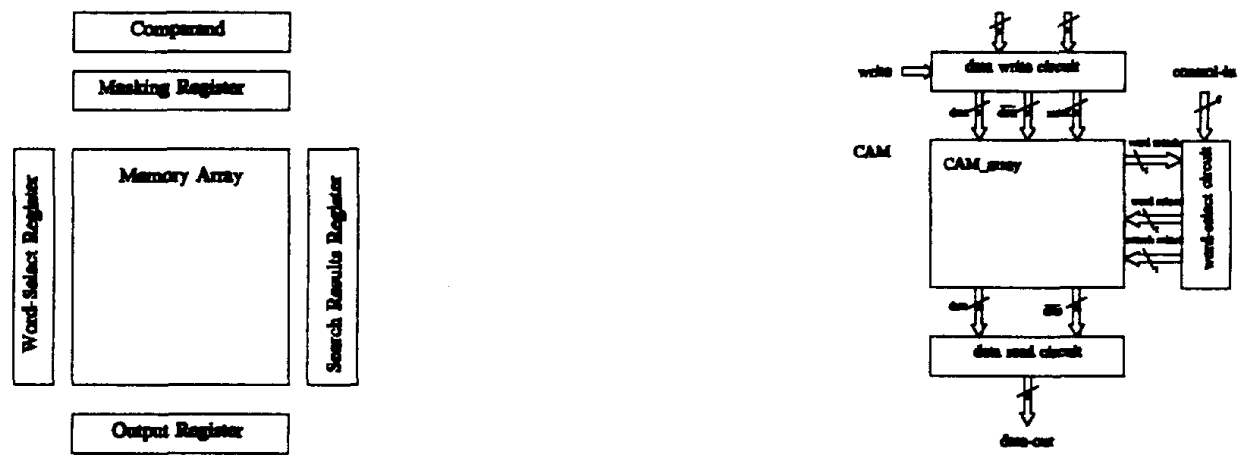

Figure 1. CAM Organization.

Figure 2. CAM Architecture.

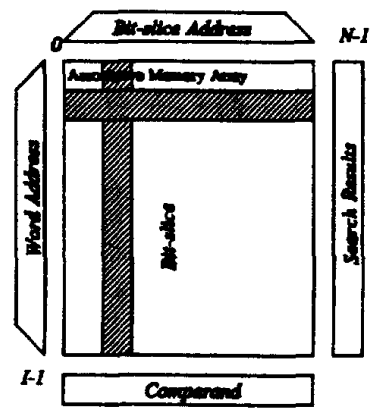

Figure 3. Bit-Serial Word-Parallel Associative Memory Organization. 


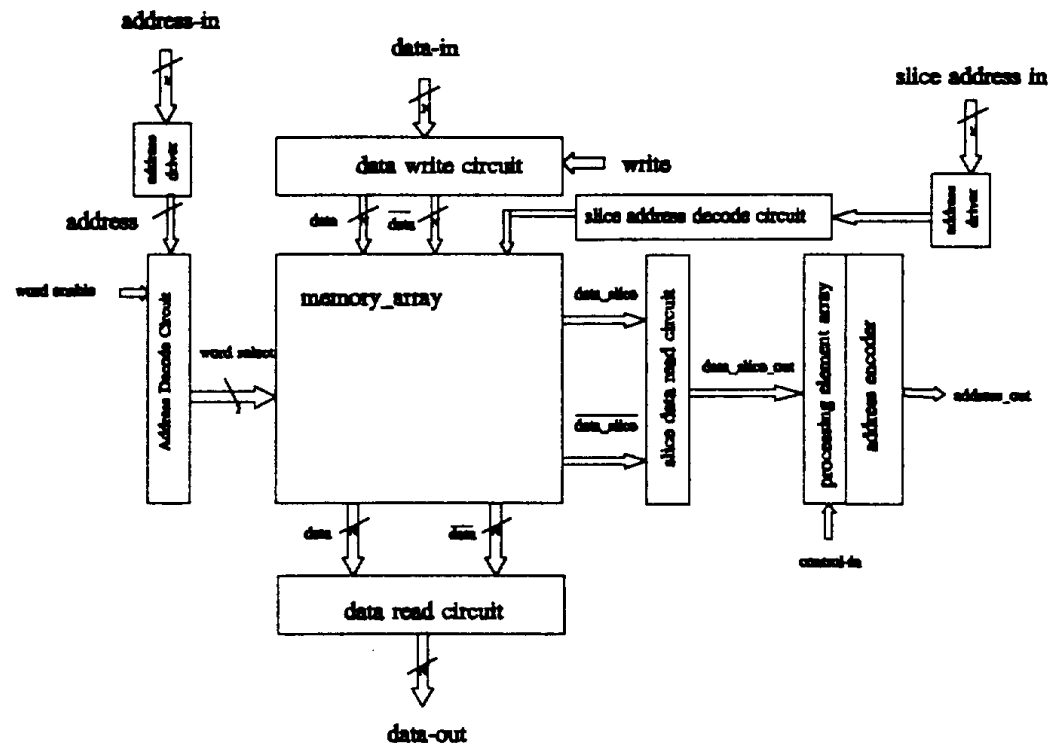

Figure 4. Bit-Serial Word-Parallel Associative Memory Architecture.

Table 1. The Number of Equivalence Searches Required to Execute Threshold Searches Upon a Database Ranging from 1 to 6 Bits in Word Length.

\begin{tabular}{||c|c|c|c|c|c|c||}
\hline & 0 searches & 1 searches & 2 searches & 3 searches & 4 searches & 5 searches \\
\hline 1-bits & 1 & 1 & 0 & 0 & 0 & 0 \\
\hline 2-bits & 1 & 3 & 0 & 0 & 0 & 0 \\
\hline 3-bits & 1 & 5 & 2 & 0 & 0 & 0 \\
\hline 4-bits & 1 & 7 & 6 & 2 & 0 & 0 \\
\hline 5-bits & 1 & 9 & 12 & 8 & 2 & 0 \\
\hline 6-bits & 1 & 11 & 20 & 20 & 10 & 2 \\
\hline
\end{tabular}


Table 2. Quantitative Analysis of Associative Memories to Determine the Best Performer for an 8-bit Parameter Limit and for a Variety of Memory Organizations.

\begin{tabular}{|c|c|c|c|c|c|c|c||}
\hline $\begin{array}{c}\mathrm{T} \\
\text { bits/ } \\
\text { word }\end{array}$ & bits/word & words/phrase & bits & words/memory & $\mathrm{y}$ & $\begin{array}{c}\mathrm{t}_{\mathrm{pam}} \\
\mathrm{t}_{1}\end{array}$ & $\frac{2\left(\mathrm{~T}+\mathrm{T}_{\mathrm{vdt}}+\mathrm{T}_{\mathrm{p} \alpha}\right.}{2+(\mathrm{T}-3) \mathrm{y}}$ \\
\hline 8 & 1 & 12 & 4 & $864+24$ & 1.0 & 3.6 & 3.71 \\
\hline 16 & 1 & 6 & 3 & $432+12$ & 1.0 & 3.25 & 2.67 \\
\hline 32 & 1 & 3 & 2 & $216+6$ & 1.0 & 2.78 & 2.26 \\
\hline 48 & 1 & 2 & 1 & $144+4$ & 1.0 & 2.44 & 2.13 \\
\hline 96 & 1 & 1 & 0 & $72+2$ & 1.0 & 1.85 & 2.04 \\
\hline
\end{tabular}

Table 3. Quantitative Analysis of Associative Memories to Determine the Best Performer for a 32-bit Parameter Limit and for a Variety of Memory Organizations.

\begin{tabular}{|c|c|c|c|c|c|c|c|}
\hline $\begin{array}{c}\mathrm{T} \\
\text { bits/ } \\
\text { word }\end{array}$ & $\begin{array}{c}T_{\text {vut }} \\
\text { bit/word }\end{array}$ & words/phrase & $\begin{array}{c}\mathrm{T}_{\mathrm{pt}} \\
\text { bits/word }\end{array}$ & words/memory & y & $\begin{array}{l}\frac{t_{c a m}}{t_{1}} \\
\leq\end{array}$ & $\frac{2\left(T+T_{v d f}+T_{p f}\right)}{2+(T-3) y}$ \\
\hline 32 & 1 & 12 & 4 & $864+24$ & 1.0 & 3.41 & 2.39 \\
\hline 64 & 1 & 6 & 3 & $432+12$ & 1.0 & 2.93 & 2.16 \\
\hline 128 & 1 & 3 & 2 & $216+6$ & 1.0 & 2.28 & 2.06 \\
\hline 192 & 1 & 2 & 1 & $144+4$ & 1.0 & 1.91 & 2.03 \\
\hline 384 & 1 & 1 & 0 & $72+2$ & 1.0 & 1.41 & 2.01 \\
\hline
\end{tabular}

